\title{
Binding of Hydrophobic Counterions by Polyelectrolyte and Their Hydrophobic Association around Polyion
}

\author{
Tomoyuki Itaya, Kazuyoshi Ueda, Hiroshi OchiaI, \\ and Akira Imamura \\ Department of Chemistry, Faculty of Science, \\ Hiroshima University, Kagamiyama, \\ Higashi-Hiroshima, Hiroshima 724, Japan
}

(Received July 17, 1992)

\begin{abstract}
The binding of $p$-ethylbenzenesulfonate ions (EBS ${ }^{-}$'s) by poly(allylammonium) cation $\left(\mathrm{PAAH}^{+}\right)$and their hydrophobic association around $\mathrm{PAAH}^{+}$have been investigated in aqueous solutions through ${ }^{1} \mathrm{H} \mathrm{NMR}$ and $\mathrm{Cl}^{-}$activity measurements. At a constant $\mathrm{PAAH}^{+} \mathrm{Cl}^{-}$ concentration, all ${ }^{1} \mathrm{H}$ NMR signals of $\mathrm{EBS}^{-}$exhibited up-field shift (ring current shift) with the increase in the NaEBS concentration. However, any chemical shift change did not observe in each signal of $\mathrm{PAAH}^{+}$. These results show that EBS ${ }^{-}$'s atmospherically bound to $\mathrm{PAAH}^{+}$associate each other due to their hydrophobic interaction. The association of $\mathrm{EBS}^{-}$'s is depressed by the decrease in the charge density of the polyion and broken by the addition of another simple salt $(\mathrm{NaCl})$ to the $\mathrm{PAAH}^{+} \mathrm{Cl}^{-} / \mathrm{NaEBS}$ system. Moreover, it was found that the counterion exchange from $\mathrm{Cl}^{-}$'s to $\mathrm{EBS}^{- \text {'s }}$ around $\mathrm{PAAH}^{+}$is efficiently promoted by the association of EBS ${ }^{- \text {'s. }}$

KEY WORDS Poly(allylammonium) Cation / Hydrophobic Counterion /

Atmospherical Binding / Association of Hydrophobic Counterions /

Counterion Exchange /
\end{abstract}

The properties of polyelectrolyte solutions depend, very much, on the electrostatic interactions between charges fixed along the polymer backbone. These interactions are also very sensitive to the presence of counterions. The addition of electrolyte to the medium screens out the repulsive interactions between charges through the counterion binding and/or condensation around the polyion with a high electrostatic potential. This counterion binding (condensation) is an important feature of polyelectrolyte solutions. ${ }^{1}$ Thus, the solution properties are considerably modified accompanying the changes in the chain conformation, water structure, degree of hydration around the polyion, and so on. On the one hand, when hydrophobic salt is used as an added electrolyte, ${ }^{2-4}$ the hydrophobic counterion, which has both hydrophilic and hydrophobic moieties, may have a different influence upon these electrostatic interactions. Especially, interactions among hydrophobic counterions should be also taken into account when polyion/hydrophobic counterion system is examined, although this interaction would be negligible in the absence of polyion. ${ }^{5}$

In the previous work, ${ }^{6}$ the interaction of three hydrophobic ions, i.e., benzenesulfonate $\left(\mathrm{BS}^{-}\right), p$-methylbenzenesulfonate $\left(\mathrm{MBS}^{-}\right), p$ ethylbenzenesulfonate $\left(\mathrm{EBS}^{-}\right)$with $\mathrm{PAAH}^{+}$ cation was investigated by ${ }^{1} \mathrm{H}$ NMR and viscosity measurements. It was found that EBS $^{-}$'s accumulated electrostatically around the polyion can associate each other through their hydrophobic interaction. But, $\mathrm{BS}^{-}$and $\mathrm{MBS}^{-}$, which are slightly less hydrophobic than $\mathrm{EBS}^{-}$, did not show any sign of such hydrophobic association. This indicates that the hydrophobicity of counterion plays an important role in the association of counterions 
around polyion. However, the mutual relation of the attractive interaction between polyion and counterions, the repulsive interaction between charges along the chain or counterions, and the hydrophobic interaction between counterions has not been cleared in details yet.

Under these circumstances, we present here a contribution to the understanding of the binding and/or association of hydrophobic counterions around the polyion. We pursued ${ }^{1} \mathrm{H} \mathrm{NMR}$ and $\mathrm{Cl}^{-}$activity measurements on the poly(allylammonium) cation/EBS ${ }^{-}$system in order to elucidate the effects of the charge density of the polyion and the coexistence of another simple salt $(\mathrm{NaCl})$ on these binding and/or association of hydrophobic counterions around the polyion.

\section{EXPERIMENTAL}

\section{Materials}

Poly(allylammonium) chloride $\left(\mathrm{PAAH}^{+} \mathrm{Cl}^{-}\right)$ used in this study was obtained from Nitto Boseki Co., Ltd. It was purified by reprecipitation with methanol contained $0.5 \mathrm{M} \mathrm{HCl}$ and dried in vacuo at room temperature for one week. ${ }^{7}$ The weight-average molecular weight of $\mathrm{PAAH}^{+} \mathrm{Cl}^{-}$was determined by light scattering to be $1.0 \times 10^{5}$ in $0.2 \mathrm{M} \mathrm{NaCl}$. Sodium $p$-ethylbenzenesulfonate (NaEBS) of analytical grade was purchased from Tokyo Kasei Co., Ltd., which was used without further purification. $\mathrm{D}_{2} \mathrm{O}(99.9 \%)$ was obtained from Matheson, U.S.A. Company. NaOD (99.5\%, Merck) was used to control the neutralization $\alpha$ of $\mathrm{PAAH}^{+}$. For chloride ion activity measurement, deionized and doubly distilled water was used as solvent. Concentrations were expressed in $C_{\mathrm{p}}$ (monomer $\mathrm{moldm}^{-3}$ ) for polymer and $C_{\mathrm{s}}\left(\mathrm{mol} \mathrm{dm}^{-3}\right)$ for salts.

\section{Measurements}

${ }^{1} \mathrm{H}$ NMR experiments were performed at $270 \mathrm{MHz}$ on a JEOL GSX270 spectrometer in pulse Fourier Transform mode (Instrument Center for Chemical Analysis, Hiroshima
University). The measurements were carried out at $27 \pm 1{ }^{\circ} \mathrm{C}$ in $\mathrm{D}_{2} \mathrm{O}(99.9 \%)$. Chemical shift was given in ppm relative to the external tetramethylsilane (TMS) standard.

Chloride ion activity measurement was carried out on an Orion 940 ion meter equipped with a chloride ion selective electrode (Orion model 94 17B) and a double junction reference electrode (Orion model 90 02). Potential measurements were performed at a constant temperature $\left(25 \pm 0.1^{\circ} \mathrm{C}\right)$ under nitrogen atmosphere. Calibration curves for $\mathrm{Cl}^{-}$ion were determined before each data set with standard solutions of sodium chloride. All potentials were taken after a constant readings were obtained (20-30 minutes).

\section{RESULTS AND DISCUSSION}

\section{Binding of $\mathrm{EBS}^{-}$'s by $\mathrm{PAAH} \mathrm{H}^{+}$and Their Hydrophobic Association around $P A A H^{+}$}

Two models are generally accepted for the interaction between polyion and hydrophobic counterions. ${ }^{8}$ One of them is the electrostatic interaction and the other is the hydrophobic interaction between the non-polar moieties of the polyion and the bound counterions. The interaction between polyion and hydrophobic counterions can be successfully probed by NMR spectroscopy. Figure 1 shows the NaEBS concentration dependence of the ${ }^{1} \mathrm{H}$ NMR spectra of the PAAH ${ }^{+} \mathrm{Cl}^{-}\left(C_{\mathrm{p}}=1.17 \times\right.$ $10^{-2} \mathrm{M}$ )-NaEBS system in $\mathrm{D}_{2} \mathrm{O}$ solution. Any chemical shift change was not observed in each signal of $\mathrm{PAAH}^{+}$. This result indicates that $\mathrm{EBS}^{-}$'s are bound to the polyion not so as to give any change in the magnetic environment of polyion protons. Accordingly, it seems that the interaction between $\mathrm{PAAH}^{+}$and $\mathrm{EBS}^{-}$'s is neither the direct hydrophobic interaction nor the strong electrostatic interaction such as site binding. Because, upon the above interactions, ${ }^{1} \mathrm{H}$ chemical shift of the polyion protons should be changed. Probably, the interaction between $\mathrm{PAAH}^{+}$and $\mathrm{EBS}^{-}$'s may be such an atmospheric binding as interposing some 


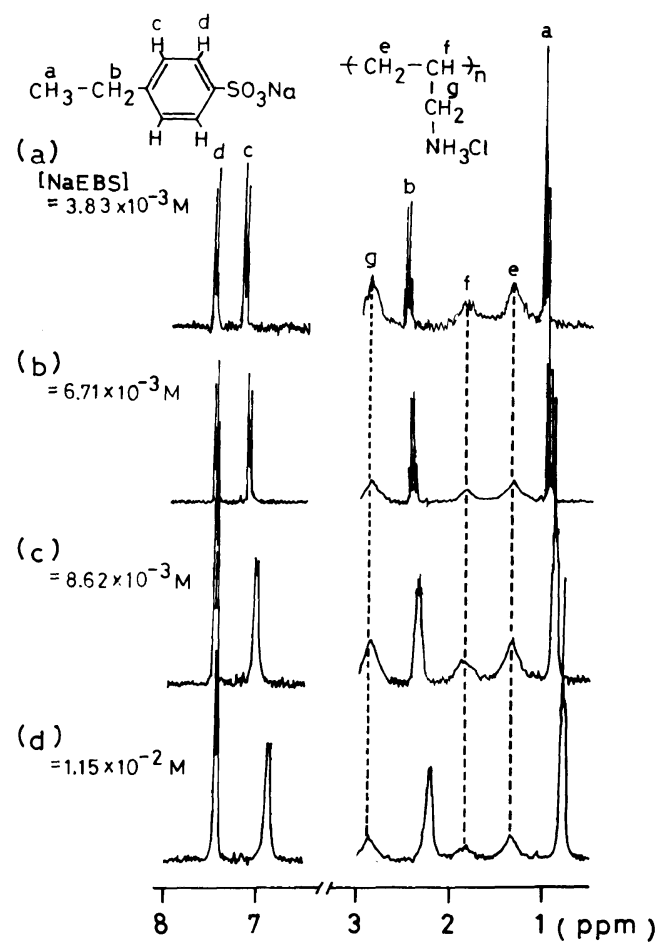

Figure 1. NaEBS concentration dependence of the ${ }^{1} \mathrm{H}$ NMR spectra of $\mathrm{NaEBS}$ and $\mathrm{PAAH}^{+} \mathrm{Cl}^{-}$in $\mathrm{D}_{2} \mathrm{O}$ solution. $\left[\mathrm{PAAH}^{+} \mathrm{Cl}^{-}\right]=1.17 \times 10^{-2} \mathrm{M}$.

hydration layers between $\mathrm{PAAH}^{+}$moieties and $\mathrm{EBS}^{-}$'s. The presence of such hydration layer between the charged backbone and the electrostatically accumulated counterions is verified from the recent neutron and X-ray scattering studies on poly(methacrylic acid) solution. ${ }^{9}$

On the other hand, each signal of EBS ${ }^{-}$ exhibited an up-field shift with increasing concentration of NaEBS. In addition, a line broadening of $\mathrm{EBS}^{-}$proton signals was observed at the higher concentrations of NaEBS $\left(C_{\mathrm{s}}=8.62 \times 10^{-3} \mathrm{M}\right.$ and $\left.C_{\mathrm{s}}=1.15 \times 10^{-2} \mathrm{M}\right)$. Similar up-field shift and line broadening have never been observed in the systems containing less hydrophobic counterions: $\mathrm{PAAH}^{+} \mathrm{Cl}^{-} / p$ methylbenzenesulfonate, $\mathrm{PAAH}^{+} \mathrm{Cl}^{-} /$benzenesulfonate and $\mathrm{PAAH}^{+} \mathrm{Cl}^{-} / n$-butanesulfonate. ${ }^{6}$ These results indicate that the up-field shift of $\mathrm{EBS}^{-}$signals is due to the inter-

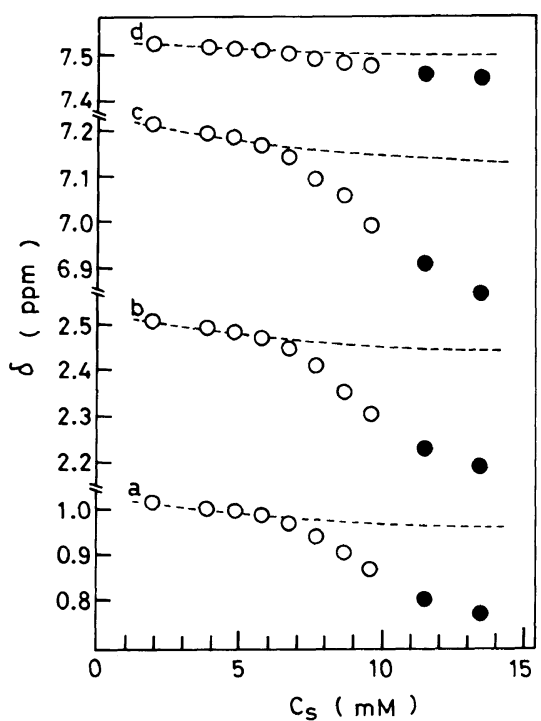

Figure 2. NaEBS concentration dependence of ${ }^{1} \mathrm{H}$ chemical shift of NaEBS in $\mathrm{D}_{2} \mathrm{O}$ solution containing $1.17 \times 10^{-2} \mathrm{M} \mathrm{PAAH}^{+} \mathrm{Cl}^{-}$. The small letters indicate a, methyl-; b, methylene-; c, meta-; and d, ortho protons. The solid symbols indicate a turbid solution. Dashed lines show the calculated values, where $\delta_{\mathrm{m}}=1.02, \delta_{\mathrm{d}}=0.80$ for methyl proton, $\delta_{\mathrm{m}}=2.51, \delta_{\mathrm{d}}=2.25$ for methylene proton, $\delta_{\mathrm{m}}=$ $7.21, \delta_{\mathrm{d}}=6.92$ for meta-proton, $\delta_{\mathrm{m}}=7.52, \delta_{\mathrm{d}}=7.45$ for ortho-proton and $K=20$.

molecular ring current effect of benzene rings of associated $\mathrm{EBS}^{-}$'s around $\mathrm{PAAH}^{+}$rather than the binding of $\mathrm{EBS}^{-}$'s to $\mathrm{PAAH}^{+}$. In addition, it can be presumed that the stacking interaction between benzene rings and the hydrophobicity of counterion play an important part in the association of counterions around the polyion. In the absence of PAAH ${ }^{+} \mathrm{Cl}^{-}$, the sharp ${ }^{1} \mathrm{H}$ NMR signals of EBS $^{-}$were observed, showing that EBS ${ }^{-}$itself does not exhibit self-association in pure water. Accordingly, it is suggested that the association of $\mathrm{EBS}^{-}$'s is induced by the presence of $\mathrm{PAAH}^{+} \mathrm{Cl}^{-}$. And, EBS ${ }^{-}$'s associate each other due to their hydrophobic interaction including the stacking interaction around $\mathrm{PAAH}^{+}$.

Figure 2 shows how the ${ }^{1} \mathrm{H}$ chemical shift of $\mathrm{EBS}^{-}$in the $\mathrm{PAAH}^{+} \mathrm{Cl}^{-} / \mathrm{NaEBS}$ system varies with the NaEBS concentration. Dotted curves represent the results calculated by a 
simple equilibrium model (this is discussed in detail later). The change of ${ }^{1} \mathrm{H}$ chemical shift of $\mathrm{EBS}^{-}$is small at low concentration of NaEBS, but it becomes very large with increasing the NaEBS concentration. The precipitation of the polymer also takes place at higher NaEBS concentrations. The accumulation of $\mathrm{EBS}^{-}$around the polyion with increasing the NaEBS concentration leads to the favorable hydrophobic association of

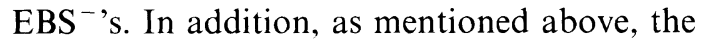
hydrophilic envelope $\left(\mathrm{NH}_{3}{ }^{+}\right.$groups) of $\mathrm{PAAH}^{+}$atmospherically interacts with the ionic head $\left(\mathrm{SO}_{3}{ }^{-}\right.$group) of the counterion directing its hydrophobic tail to the outside. ${ }^{10}$ Therefore, the environments around $\mathrm{PAAH}^{+}$ become more hydrophobic as a whole, which leads to precipitation.

The order of magnitude of the ring current shift is $m$-proton $>$ methylene proton $>$ methyl proton $>o$-proton. The change of the chemical shift of $o$-proton is fairly smaller than that of other protons. The magnitude and order of the ring current shift are highly dependent on the mutual orientation of the aromatic ring planes of the associated $\mathrm{EBS}^{-}$'s. As is shown in the previous paper, ${ }^{6}$ the structure of associated EBS $^{-}$'s is assumed as follows. The aromatic rings are overlapped each other, skewing by about $60^{\circ}$ due to the charge repulsion of sulfonate groups and the steric hindrance of ethyl groups. The smaller change in chemical shift of $o$-proton may be attributable to the effect of the magnetic anisotropy of $\mathrm{S}=\mathrm{O}$ double bond in the sulfonate group, which probably leads to a rather decreased magnetic shielding. ${ }^{11}$

\section{Effect of the Charge Density of Polyion on the Association of $E_{B S^{-}}$s}

Figure 3 shows the effect of the charge density of the polyion on the ${ }^{1} \mathrm{H}$ chemical shift of $\mathrm{EBS}^{-}$in $\mathrm{PAAH}^{+} \mathrm{Cl}^{-} / \mathrm{NaEBS}$ system. The abscissa in Figure 3 represents the degree of neutralization $\alpha$ of $\mathrm{PAAH}^{+}\left(-\mathrm{NH}_{3}{ }^{+} \rightarrow\right.$ $\left.-\mathrm{NH}_{2}+\mathrm{H}^{+}\right)$. The counterion binding to poly-

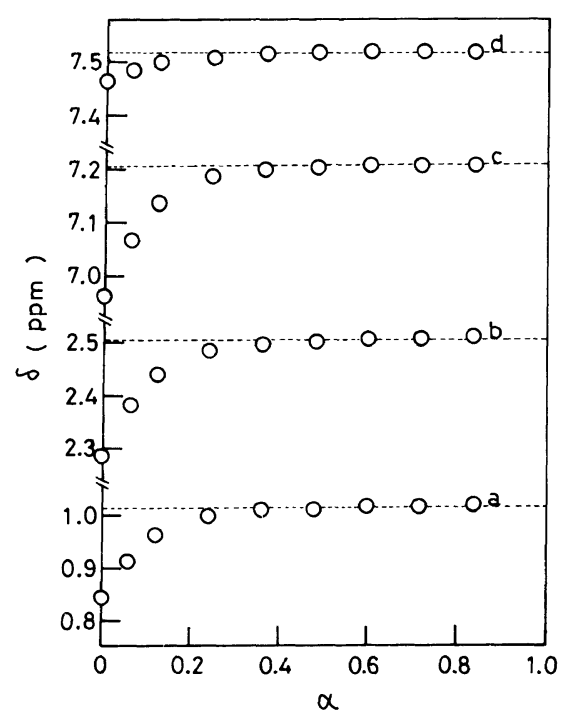

Figure 3. The effect of the charge density of $\mathrm{PAAH}^{+}$on the ${ }^{1} \mathrm{H}$ chemical shift of $8.92 \times 10^{-3} \mathrm{M}$ NaEBS in the presence of $1.17 \times 10^{-2} \mathrm{M} \mathrm{PAAH}^{+} \mathrm{Cl}^{-}$. The small letters indicate a, methyl-; b, methylene-; c, meta-; and d, ortho- protons.

ion depends on its charge density. ${ }^{12}$ At $\alpha=0$, $\mathrm{PAAH}^{+}$is a fully charged vinylic polyelectrolyte forming a high electrostatic field around it. Therefore, the added $\mathrm{EBS}^{-}$'s are accumulated around the $\mathrm{PAAH}^{+}$moieties. This accumulation of the counterions by the polyion induces the hydrophobic association of the counterions. However, as the charge density of the polyion decreases ( $\alpha$ increases), ${ }^{1} \mathrm{H}$ signals of $\mathrm{EBS}^{-}$exhibit a down-field shift from the higher position at $\alpha=0$. And, even at $\alpha=0.4$, they shift back to each original position as in the absence of $\mathrm{PAAH}^{+} \mathrm{Cl}^{-}$. This result shows that the association of $\mathrm{EBS}^{-}$'s is not induced under the condition that the charge density of the polyion is low (for example $\alpha>0.4$ ). On the other hand, according to Mannings's counterion condensation theory, ${ }^{13}$ since the charge density parameter $\xi$ is 1.71 at $\alpha=0.4$, some counterions should be condensed around the polyion. However, the present experimental result suggests that even if the counterions exist in high concentration around the polyion, their mutual interaction seems to 


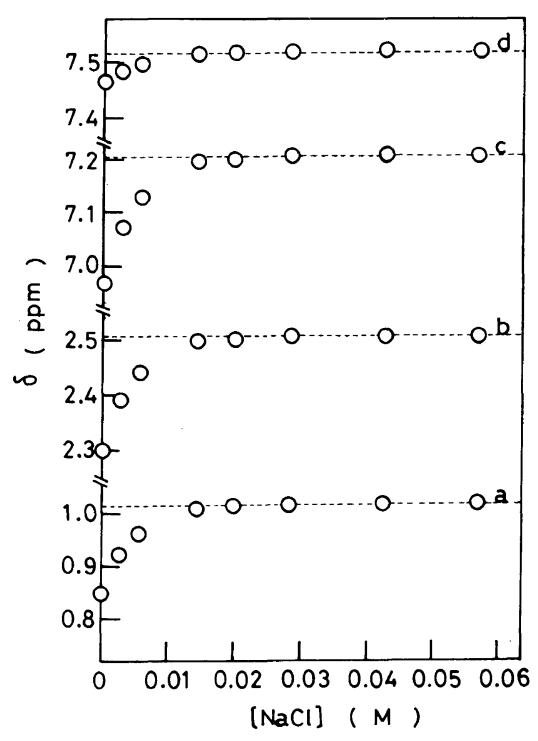

Figure 4. The effect of $\mathrm{NaCl}$ on the ${ }^{1} \mathrm{H}$ chemical shift of $8.92 \times 10^{-3} \mathrm{M}$ NaEBS in the presence of $1.17 \times 10^{-2} \mathrm{M}$ $\mathrm{PAAH}^{+} \mathrm{Cl}^{-}$. The small letters indicate a, methyl-; b, methylene-; c, meta- and d, ortho- protons.

be nil at $\alpha=0.4$.

Effect of the Added Salt $(\mathrm{NaCl})$ on the Association of $E B S^{-}$'s

Figure 4 shows the effect of added $\mathrm{NaCl}$ on the ${ }^{1} \mathrm{H}$ chemical shift of EBS ${ }^{-}$in the presence of $\mathrm{PAAH}^{+} \mathrm{Cl}^{-}$. When $\mathrm{NaCl}$ is added to the PAAH ${ }^{+} \mathrm{Cl}^{-} / \mathrm{NaEBS}$ system, ${ }^{1} \mathrm{H}$ signals of $\mathrm{EBS}^{-}$shift to the low magnetic field and on further addition of $\mathrm{NaCl}$ they shift back to the original position as in the absence of $\mathrm{PAAH}^{+} \mathrm{Cl}^{-}$. This result shows that the association of $\mathrm{EBS}^{-}$'s is broken by the added $\mathrm{Cl}^{-}$'s. The charges on the polyion are shielded by an excess of $\mathrm{NaCl}$ approached to the polyion. Association of EBS ${ }^{-}$'s is depressed as a result of the shielding of charges on the polyion. Concurrently, EBS ${ }^{-}$'s are preferentially removed from the $\mathrm{PAAH}^{+}$moieties by $\mathrm{Cl}^{-}$'s. Until now, the counterion binding to polyion has been interpreted in terms of the size of counterion ${ }^{14}$ and/or the state of hydration of both polyion and counterions. ${ }^{15,16}$ The affinity of bulky and hydro-

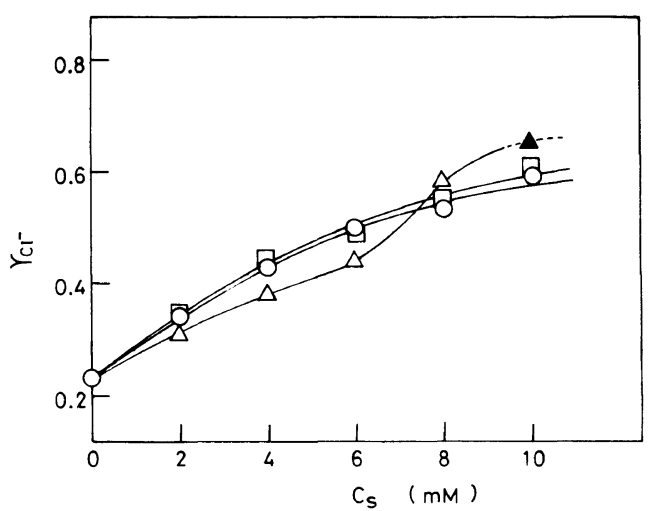

Figure 5. The dependence of the activity coefficient of $\mathrm{Cl}^{-}$for $1.05 \times 10^{-2} \mathrm{M} \mathrm{PAAH}{ }^{+} \mathrm{Cl}^{-}$on the hydrophobic salt concentration. ( $O$ ), NaBS; $(\square)$, NaMBS; $(\triangle)$, NaEBS. The solid symbol indicates a turbid solution.

phobic EBS ${ }^{-}$to the hydrophilic polymer such as $\mathrm{PAAH}^{+}$seems to be much less than $\mathrm{Cl}^{-}$. Therefore, when amounts of NaEBS added to the aqueous $\mathrm{PAAH}^{+} \mathrm{Cl}^{-}$(a constant concentration) solution without $\mathrm{NaCl}$ are relative small, many $\mathrm{Cl}^{-}$'s coexisting prevent an effective approach of $\mathrm{EBS}^{-}$'s to the polyion moieties. However, large amounts of NaEBS ia added to the solution, the association of EBS $^{-}$'s is subject to occur. The associated EBS $^{-}$'s has a property of di- and/or multivalent counterion and strongly interacts with $\mathrm{PAAH}^{+}$. Consequently, counterion exchange from $\mathrm{Cl}^{-}$'s to $\mathrm{EBS}^{-}$'s around $\mathrm{PAAH}^{+}$may be much promoted. In order to obtain the information about this counterion exchange around $\mathrm{PAAH}^{+}$, we measured the activity of $\mathrm{Cl}^{-}$for $\mathrm{PAAH}^{+} \mathrm{Cl}^{-}$in hydrophobic salt solutions.

The Activity Measurements of Chloride Ion for

$\mathrm{PAAH}^{+} \mathrm{Cl}^{-}$in Hydrophobic Salt Solutions

Figure 5 shows the dependence of the activity coefficient of $\mathrm{Cl}^{-}\left(\gamma_{\mathrm{Cl}^{-}}\right)$upon the concentration of added hydrophobic salts, i.e., sodium benzenesulfonate ( $\mathrm{NaBS}$ ), sodium $p$-methylbenzenesulfonate (NaMBS) and NaEBS at a constant $\mathrm{PAAH}^{+} \mathrm{Cl}^{-}$concentration. The comparison of the $\gamma_{\mathrm{Cl}^{-}}$value in NaEBS solution 
with that in NaBS and NaMBS solutions elucidate the counterion exchange promoted by the association of $\mathrm{EBS}^{-}$'s. In the low salt concentration range, all $\gamma_{\mathrm{Cl}^{-}}$values monotonously increase with the salt concentration. This behavior is a characteristic property of polyelectrolyte solution. ${ }^{17}$ In addition, the $\gamma_{\mathrm{Cl}^{-}}$ values decrease with the order of the hydrophobicity of the counterions. On the other hand, in the higher salt concentration range, the $\gamma_{\mathrm{Cl}^{-}}$value in the $\mathrm{PAAH}^{+} \mathrm{Cl}^{-} /$ NaEBS system abruptly increases going over the values of other two systems, and after that the solution becomes turbid. The NaEBS concentration which the $\gamma_{\mathrm{Cl}^{-}}$suddenly increased corresponds well to that ${ }^{1} \mathrm{H}$ chemical shift begins to change largely to up-field. The above result implies that the counterion exchange from $\mathrm{Cl}^{-}$'s to $\mathrm{EBS}^{-}$'s was much promoted by the association of $\mathrm{EBS}^{-}$'s, causing an abrupt increase in the $\gamma_{\mathrm{Cl}^{-}}$value.

Generally, the stronger the interaction of an added counterion with a polyion, the higher the activity of coexisting $\mathrm{Cl}^{-}$. When $\mathrm{EBS}^{-}$'s associate each other around the polyion, the associated $\mathrm{EBS}^{-}$'s have a character of divalent and/or multivalent counterion. Consequently, they strongly interact with $\mathrm{PAAH}^{+}$and preferentially remove $\mathrm{Cl}^{-}$'s from the polyion moieties. At the same time, the ionic hydration around the charged groups on the polyion may decrease by the hydrophobic groups of EBS ${ }^{-}$, which leads to the precipitation of the polyion.

Analysis of Association Behavior of $E_{B S^{-}}$'s around $\mathrm{PAAH}^{+}$by Simple Equilibrium Model

As shown in Figure $1,{ }^{1} \mathrm{H}$ NMR signals of $\mathrm{EBS}^{-}$shift to up-field due to the association of $\mathrm{EBS}^{- \text {'s }}$ around $\mathrm{PAAH}^{+}$. Moreover, the line broadening was observed in each proton of $\mathrm{EBS}^{-}$, especially in methylene proton. This broadening suggests that the motion of the methylene protons is highly restricted by the

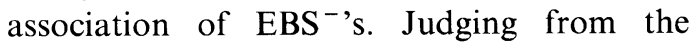
change of above ${ }^{1} \mathrm{H}$ NMR signals of $\mathrm{EBS}^{-}$, it seems that a rapid exchange is set up between the free monomer and aggregate (dimer) of $\mathrm{EBS}^{-}$around $\mathrm{PAAH}^{+}$. Accordingly, the higher fraction of associated $\mathrm{EBS}^{-}$ions, the larger this change of ${ }^{1} \mathrm{H}$ NMR signals.

It is of interest to analyse the observed chemical shift of $\mathrm{PAAH}^{+} \mathrm{Cl}^{-} / \mathrm{NaEBS}$ system in terms of a rapid exchange between the free monomer and dimer of $\mathrm{EBS}^{-}$around $\mathrm{PAAH}^{+}$. The following simple equilibrium was adopted under the condition that the concentration of $\mathrm{PAAH}^{+} \mathrm{Cl}^{-}$is constant.

$$
\begin{gathered}
2 \cdot \text { monomer } \rightleftharpoons \text { dimer }\left(\text { around } \mathrm{PAAH}^{+}\right)( \\
K=[\mathrm{D}] /[\mathrm{M}]^{2}
\end{gathered}
$$

where [M] and [D] represent the molar concentrations of the monomer and dimer forms, respectively. Then, the chemical shift, $\delta$, can be expressed by a weight average of the chemical shifts for two forms.

$$
\delta=X_{\mathrm{m}} \delta_{\mathrm{m}}+X_{\mathrm{d}} \delta_{\mathrm{d}}
$$

where $\delta_{\mathrm{m}}$ and $\delta_{\mathrm{d}}$ denote the respective chemical shifts of the monomer and dimer forms with $X_{\mathrm{m}}$ and $X_{\mathrm{d}}$ being the corresponding molar fractions of $\mathrm{EBS}^{-}$in the two forms $\left(X_{\mathrm{m}}+\right.$ $\left.X_{\mathrm{d}}=1\right)$.

From the eq 2 and 3, the chemical shift can be expressed by

$$
\begin{aligned}
\delta= & \delta_{\mathrm{d}}+\left(\delta_{\mathrm{d}}-\delta_{\mathrm{m}}\right) \times \\
& \left\{1-\left(1+8 K\left[\mathrm{EBS}^{-}\right]_{t}\right)^{1 / 2}\right\} /\left(4 K\left[\mathrm{EBS}^{-}\right]_{t}\right)
\end{aligned}
$$

where $\left[\mathrm{EBS}^{-}\right]_{t}$ is the total concentration of $\mathrm{NaEBS}$ and $\left[\mathrm{EBS}^{-}\right]_{t}=[M]+2[\mathrm{D}]$.

To determine the $K$ and $\delta_{\mathrm{d}}$ 's which can reproduce the experimental results (the solid symbols in Figure 2), an iterative procedure was adopted, where the values of $\delta_{\mathrm{m}}$ 's were taken as those in the absence of $\mathrm{PAAH}^{+} \mathrm{Cl}^{-}$. However, any pair of $K$ and $\delta_{\mathrm{d}}$ 's values covering adequately experimental results over the whole concentration range of NaEBS examined could not be obtained. For example, the dotted curves in Figure 2 represent the calculated results using the values of $K$ and $\delta_{\mathrm{d}}$ 's shown in Figure 2. In the lower NaEBS 


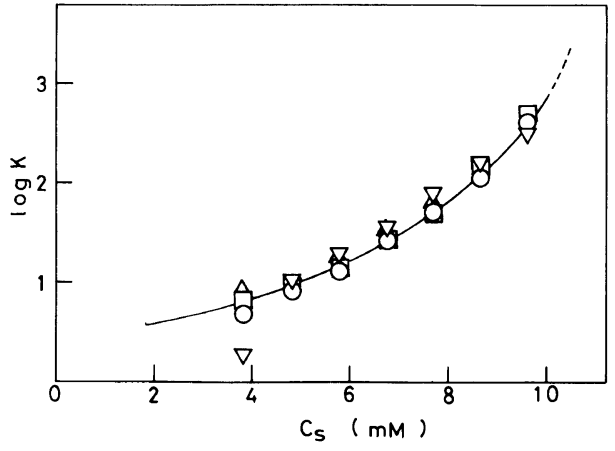

Figure 6. The dependence of the association constant $K$ of EBS $^{-}$ions around PAAH ${ }^{+}$on the NaEBS concentration. The sysmbols indicate the values calculated for $(\triangle)$ methyl-, ( $\square)$ methylene-, (O) meta- and $(\nabla)$ ortho-protons.

concentration range, the calculated values agree well with the experimental data, but the deviation from the experimental values becomes large at higher NaEBS concentrations. This implies that the simple equilibrium model (eq 1) is inapplicable to the present experimental data at higher NaEBS concentrations. Multiple aggregates such as trimer and tetramer may be formed under the condition that the local concentration of $\mathrm{EBS}^{-}$around $\mathrm{PAAH}^{+}$is very high. In addition, since the $\mathrm{EBS}^{-}$'s are accumulated in high concentration around $\mathrm{PAAH}^{+}$, the association of $\mathrm{EBS}^{- \text {' }} \mathrm{s}$ may be cooperatively enhanced.

Thus, to clarify the cooperative association of $\mathrm{EBS}^{-}$'s around the polyion, it is worthwhile to examine the change of $K$ with the NaEBS concentration. Using eq 2,3 , and 4 , the value of $K$ was evaluated from the observed NaEBS concentration dependence of each proton signal, and is shown in Figure 6 (for $d_{m}$ 's and $\delta_{\mathrm{d}}$ 's, the values shown in caption of Figure 2 were used). The value of $K$ rapidly increases, as expected, with the increase in the NaEBS concentration. This strongly suggests the cooperative association of $\mathrm{EBS}^{- \text {' }}$ s around the polyion, i.e., an increase in the local concentration of $\mathrm{EBS}^{-}$considerably enhances the association. Furthermore, it should be noted that all the $K$ values calculated from different proton signals are converged on a single smoothed curve. This also may give a powerful collaboration of our assertion that the observed chemical shifts of $\mathrm{EBS}^{-}$in the presence of PAAH ${ }^{+} \mathrm{Cl}^{-}$are originated in a single process, that is, the ring current effect due to the association of $\mathrm{EBS}^{-}$'s.

\section{CONCLUSION}

In this study, EBS $^{-}$'s found to be atmospherically bound by $\mathrm{PAAH}^{+} \mathrm{Cl}^{-}$and to associate each other around $\mathrm{PAAH}^{+}$through their hydrophobic interaction. Since the association occurs in the presence of $\mathrm{PAAH}^{+}$, it is influenced by the charge density of the polyion and the coexisting small ions $\left(\mathrm{Cl}^{-}\right)$. From a simple equilibrium model, the cooperative association of $\mathrm{EBS}^{-}$'s around $\mathrm{PAAH}^{+}$was demonstrated. In addition, as a result of the association of $\mathrm{EBS}^{-}$'s, the counterion exchange from $\mathrm{Cl}^{-}$'s to $\mathrm{EBS}^{-}$'s around $\mathrm{PAAH}^{+}$is efficiently promoted. At the same time, the ionic hydration of charged groups on the polyion may decrease by the hydrophobic groups of $\mathrm{EBS}^{-}$, which leads to the precipitation of the polyion.

\section{REFERENCES}

1. F. Oosawa, "Polyelectrolytes," Marcel Dekker, New York, N. Y., 1971

2. S. Miyamoto and N. Imai, Biophys. Chem., 11, 91 (1980).

3. M. Satoh, E. Yoda, T. Hayashi, and J. Komiyama, Macromolecules, 24, 1123 (1991).

4. N. J. Turro, T. Okubo, C.-J. Chung, J. Emert, and R. Catena, J. Am. Chem. Soc., 104, 4799 (1982).

5. W. G. Herkstroeter, P. A. Martic, S. E. Hartman, J. L. R. Williams, and S. Farid, J. Polym. Sci., Polym. Chem. Ed., 21, 2473 (1983).

6. T. Itaya, K. Ueda, and H. Ochiai, Polym. J., 24, 539 (1992).

7. H. Ochiai, M. Handa, H. Matsumoto, T. Moriga, and I. Murakami, Macromol. Chem., 186, 2547 (1985).

8. N. J. Turro and I. F. Pierrola, J. Phys. Chem., 87, 2420 (1983). 
9. J. Plestil, Yu. M. Ostanevich, Yu. V. Bezzabotnov, and D. Hlavata, Polymer, 27, 1241 (1986).

10. T. Itaya and H. Ochiai, Polym. Commun., 32, 564 (1991).

11. J. G. Pritchard and P. C. Lauterber, J. Am. Chem. Soc., 83, 2105 (1961).

12. M. Nagasawa and I. Kagawa, J. Polym. Sci., 25, 61 (1957).

13. G. S. Manning, J. Chem. Phys., 51, 924 (1969).
14. H. P. Gregor and M. Frederick, J. Polym. Sci., 23, 451 (1957)

15. M. Hara, A. H. Lee, and J. Wu, J. Polym. Sci., B, Polym. Phys, Ed., 25, 1407 (1987).

16. H. Ochiai, Y. Anabuki, O. Kojima, K. Tominaga, and I. Murakami, J. Polym. Sci., B, Polym. Phys. Ed., 28, 233 (1990).

17. B. Boussouria, A. Ricard, and R. Audebert, J. Polym. Sci., B, Polym. Phys. Ed., 26649 (1988). 